\title{
Towards tactile sensing applied to underwater autonomous vehicles for near shore survey and de-mining
}

\author{
T. Rooney*, A.G. Pipe, S. Dogramadzi and M.J. Pearson \\ Bristol Robotics Laboratory, University of the West of England, \\ Bristol, BS161QD, UK \\ ${ }^{*}$ E-mail: thomas.rooney@live.uwe.ac.uk \\ www.brl.ac.uk
}

\begin{abstract}
Artificial tactile whisker sensors demonstrate an approach to localisation [1] that is robust to harsh environmental disturbances, endowing autonomous systems with the ability to operate effectively in confined, noisy and visually occluded spaces, such as collapsed buildings or mine shafts, where conventional sensors become unreliable [2]. Marine engineering applications could benefit from such tactile sensors due to the lack of robust underwater close proximity sensing techniques. Animals such as walruses, seals and manatees all have exquisitely sensitive whiskers, which they use for hunting and foraging. Building upon a recent pilot study in underwater tactile sensing [3], we present the motivation for further research and our work plans toward a demonstrator platform for near shore survey and demining.

Advanced underwater operations, such as demining and maintenance of an oil rig, require intervention and surveying. A diver, or a trained animal like a seal, would intuitively use tactile perception to perform this task. A Remotely Operated Vehicle (ROV) equipped with an appropriate sensory and motor control infrastructure and, importantly, a human operator, could also perform this task. However, divers, animals and ROVs have numerous disadvantages such as risk to human life, fatigue, short operational range, the need of support vessels and ultimately cost[4]. Thus, a requirement exists for improvements to be made to current Autonomous Underwater Vehicle (AUV) technologies to allow them to operate and interact effectively in close proximity and in visually occluded environments. This is where the deployment of tactile whiskers becomes apparent. An array of such tactile sensors mounted on a mobile submersible could be used to generate a "haptic map" of a region of the work area, containing characteristic features such as surface form, texture and compliance. Inspiration for the design of such an array will come from the Walrus, which have whiskers of varying length and thickness, that are capable of distinguishing small shapes in the silt of the seabed [5]. Related robotic work in this field include the MechaLobster walking AUV [3] which demonstrated a novel test platform for a single actuated whisker to undertake preliminary underwater tactile experiments. Also of note is the robolobster[6] Where a biomemetic approach was taken to demining utilising a much simpler tactile sense. Which featured cheliped mounted tactile bump sensors with binary output of a $2 \mathrm{D}$ perception.
\end{abstract}


For an AUV to successfully complete an underwater task, we have identified that the following criteria should be satisfied:

1. The agent must maintain an accurate estimate of it's location with respect to the work area. This validates the data or interactions undertaken, feeding into a 3D world model whiskers which will insert high probability information, by means of contacting and whisking the surrounding surfaces[1].

2. The agent must identify its target area/object. By palpating and probing the target surface, additional information can be extracted such as texture and compliance, when fused with surface form, a classification of the area/object can be made.

3. The agent must interact with its environment with high precision; at the low level, whiskers will provide data to be conditioned into a usesable signal by a control system, this then provides a foundation for safe and accurate interactions.

Our ultimate goal is to build a demonstrator platform that can meet these criteria using an array of artificial whiskers. The initial goal will be to characterise the response of candidate single whisker designs to controlled marine environments in the lab and in the real world. This will constrain our preliminary signal processing algorithms and establish bench mark datasets for future reference.

Acknowledgements. This work is funded by DSTL as part of their national Ph.D. programme

Keywords: Biomimetic; Whisker; Autonmous Underwater Vehicles (AUV);

\section{References}

1. Fox, C., Evans, M., Pearson, M., Prescott, T.: Tactile SLAM with a biomimetic whiskered robot. In: IEEE International conference on Robotics and Automation (ICRA), IEEE (2012) [IN PRESS]

2. Pearson, M.J., Pipe, A.G., Melhuish, C., Mitchinson, B., Prescott, T.J.: Whiskerbot: A robotic active touch system modeled on the rat whisker sensory system. Adaptive Behavior 15 (2007) 223-240

3. Rooney, T., Pearson, M., Welsby, J., Horsfield, I., Sewell, R., Dogramadzi, S.: Object localisation using active whiskers on underwater autonomous walking robots. In: 14th International conference on Climbing and Walking Robots (CLAWAR). (2011) 190-195

4. Chance, T., Kleiner, A., Northcutt, J.: The autonomous underwater vehicle (AUV): a cost-effective alternative to deep- towed technology. Integrated Coastal Zone Management 2(7) (2000) 6569

5. Kastelein, R., van Gaalen, M.: The sensitivity of the vibrissae of a pacific walrus(odobenus rosmarus divergens). Aquatic Mammals 14(3) (1988) 123-133

6. Ayers, J.: The lobster robot. In: Weapons systems technology information analysis centre. Volume 6. (2005) 1-5 